\title{
Cilostazol induces cellular senescence and confers resistance to etoposide-induced apoptosis in articular chondrocytes
}

\author{
KANG MI KIM ${ }^{1}$, JONG MIN KIM ${ }^{2}$, YOUNG HYUN YOO ${ }^{2}$, JEUNG IL KIM ${ }^{3}$ and YOUNG CHUL PARK ${ }^{1}$ \\ ${ }^{1}$ Department of Microbiology and Immunology, Pusan National University School of Medicine, Yangsan, Gyeongnam \\ 626-870; ${ }^{2}$ Department of Anatomy and Cell Biology, Dong-A University School of Medicine, Busan 602-714; \\ ${ }^{3}$ Department of Orthopedic Surgery, Pusan National University Hospital, Busan 602-739, Republic of Korea
}

Received November 14, 2011; Accepted December 30, 2011

DOI: $10.3892 / \mathrm{ijmm} .2012 .892$

\begin{abstract}
We recently reported that cilostazol protects chondrocytes against stress-induced apoptosis and prevents cartilage destruction in an osteoarthritis (OA) model. In the present study, we elucidate the mechanism underlying the protective effect induced by cilostazol against stressinduced apoptosis in chondrocytes. Cilostazol significantly reduced the expression of type II collagen and stimulated the accumulation of $\beta$-catenin in primary rat articular chondrocytes. Moreover, cilostazol-induced chondrocytes showed induction of senescent phenotypes, such as changes in cell morphology, decrease in cell proliferation and increase in specific senescence-associated $\beta$-galactosidase (SA- $\beta$-gal) staining. Moreover, dedifferentiated chondrocytes obtained by serial subculture showed cellular senescence that increased with passage number. In addition, the percentage of terminal dUTP nick end-labeling (TUNEL)-positive cells was higher when chondrocytes were treated with cilostazol and the apoptosis inducer etoposide than when the cells were treated with etoposide alone. Our findings suggest that cilostazol induces dedifferentiation and senescence in rat articular chondrocytes and renders them resistant to etoposide-induced apoptosis.
\end{abstract}

\section{Introduction}

The stability of articular cartilage depends on the biosynthetic activities of chondrocytes, which are formed by the

Correspondence to: Dr Young Chul Park, Department of Microbiology and Immunology, Pusan National University School of Medicine, Yangsan, Gyeongnam 626-870, Republic of Korea E-mail: ycpark@pusan.ac.kr

Abbreviations: SA- $\beta$-gal, senescence-associated $\beta$-galactosidase; ECM, extracellular matrix; NO, nitric oxide; OA, osteoarthritis; TUNEL, terminal deoxynucleotidyl transferase-mediated dUTP nick end-labeling; PARP, poly(ADP-ribose) polymerase; PI, propidium iodide; ECL, enhanced chemiluminescence

Key words: cilostazol, chondrocytes, dedifferentiation, senescence, apoptosis differentiation of mesenchymal cells during embryonic development $(1,2)$. Chondrocytes are the only cells found in articular cartilage. They synthesize appropriate extracellular matrix (ECM) molecules to maintain cartilage homeostasis $(3,4)$. Cartilage ECM molecules such as type II collagen and sulfated proteoglycan play a crucial role in regulating chondrocyte functions by facilitating cell-matrix interactions (5). Loss of chondrocyte activity is associated with the degradation of articular cartilage in the cases of cartilage diseases such as osteoarthritis and rheumatoid arthritis, eventually leading to joint destruction (6-8).

Senescent cells remain metabolically active and show altered expression of regulatory proteins that regulate survival and proliferation. Cellular senescence is classified into 2 types. Intrinsic replicative senescence is associated with the changes in DNA structure and function, including progressive telomere shortening (9). In contrast, extrinsic telomere-independent senescence results from diverse stimuli, including ultraviolet radiation, oxidative stress, oncogene activation, and proinflammatory cytokines (10-12). These stimuli cause extrinsic stress-induced senescence in articular chondrocytes. Chondrocyte senescence plays an important role in aging and articular cartilage degeneration (13). Senescent chondrocytes accumulate with age in articular cartilage, and a correlation between increasing age and incidence of osteoarthritis has been noted $(9,14)$.

Cilostazol is known to increase the intracellular level of cyclic AMP by blocking its hydrolysis by phosphodiesterase type III (15). Cilostazol functions as a platelet aggregation inhibitor (15) and vasodilator (16) and is mainly used for treating patients with peripheral arterial disease (17) and intermittent claudication (18). Our recent study showed that cilostazol protects rat chondrocytes against nitric oxide (NO)-induced apoptosis and prevents cartilage destruction in a rat model of osteoarthritis (OA) (19). The apoptotic effect of cilostazol in synovial cells from rheumatoid arthritis patients has also been reported (20). However, the role of cilostazol in the development, maintenance, and degeneration of articular cartilage is not known.

We found that cilostazol reduces the levels of phenotypic markers of differentiation, such as type II collagen and induces cellular senescence in primary rat articular chondrocytes. We also showed that cilostazol-induced senescent chondrocytes are resistant to etoposide-induced apoptosis. 


\section{Materials and methods}

Reagents. Cilostazol (OPC-13013) was generously donated by Otsuka Pharmaceutical (Tokushima, Japan). Protease inhibitor cocktail, trypan blue (0.4\%), 5-bromo-4-chloro-3-indolyl $\beta$-D-galactoside (X-gal), glutaraldehyde, formaldehyde, potassium ferrocyanide, potassium ferricyanide, and etoposide were purchased from Sigma-Aldrich Chemical Co. (St. Louis, MO, USA). Fetal bovine serum (FBS), Dulbecco's modified Eagle's medium (DMEM) and other culture reagents were purchased from Hyclon (Logan, UT, USA). Anti-poly(ADP-ribose) polymerase (PARP), Bax, type II collagen, $\beta$-catenin, and $\beta$-actin antibodies were obtained from Santa Cruz Biotechnology (Santa Cruz, CA, USA). The secondary horseradish peroxidase (HRP)conjugated antibody and the enhanced chemiluminescence (ECL) Western blotting kit were obtained from Amersham Pharmacia Biotech (Piscataway, NJ, USA).

Cell culture of articular chondrocytes. Articular chondroctyes for primary culture were isolated from slices of knee joint cartilage of 5-week-old female Sprague-Dawley rats (Samtako BioKorea, Osan, Korea). Chondrocytes were isolated by enzymatic digestion for $1 \mathrm{~h}$ with $0.2 \%$ type II collagenase in DMEM. The chondrocytes were briefly centrifuged, and the cells were resuspended in DMEM supplemented with $10 \%$ heat-inactivated FBS and antibiotics (50 U/ml penicillin, $50 \mu \mathrm{g} / \mathrm{ml}$ streptomycin) at $37^{\circ} \mathrm{C}$ with $5 \% \mathrm{CO}_{2}$ in air atmosphere. Cells were plated on culture dishes at a density of $5 \times 10^{4} \mathrm{cells} / \mathrm{cm}^{2}$. The medium was replaced every 2 days, and cells reached confluence at $\sim 4-5$ days after culture; this was designated as passage 0 (P0). The $\mathrm{P} 0$ cells were serially subcultured up to P6 by plating cells at a density of $5 \times 10^{4}$ cells $/ \mathrm{cm}^{2}$.

Evaluation of cell viability. Cell viability was determined using the trypan blue exclusion assay. Chondrocytes were plated at $1 \times 10^{5}$ cells per 6-well plates and incubated for $24 \mathrm{~h}$. Cells were cultured for different times in the presence or absence of various concentrations of cilostazol in fresh DMEM medium. After incubation, the cells were washed with phosphate-buffered saline (PBS), and viable cells were scored by the trypan blue dye exclusion assay by a hemocytometer.

TUNEL assay for the detection of apoptotic cells. Cells were washed with $1 \%$ PBS/BSA and fixed in $4 \%$ paraformaldehyde for 15 min. Next, they were washed with PBS/BSA and permeabilized in $0.1 \%$ Triton-X 100 for $5 \mathrm{~min}$ on ice. Fluorescein isothiocyanate (FITC)-conjugated dUTP was used for the terminal deoxynucleotidyl transferase-mediated dUTP nick end-labeling (TUNEL) assay that was performed using the Apoptosis Detection System kit (Roche Molecular Biochemicals, Mannheim, Germany) according to the manufacturer's instructions.

Senescence-associated $\beta$-galactosidase (SA- $\beta$-gal) staining assay. SA- $\beta$-gal staining assay was performed at $\mathrm{pH} 6.0$ as described by Dimri et al, with a modification (21). Cells were washed in PBS, fixed for $5 \mathrm{~min}$ (room temperature) in $0.2 \%$ glutaraldehyde $/ 2 \%$ formaldehyde, washed in PBS, and incubated with SA- $\beta$-gal stain solution $(1 \mathrm{mg} / \mathrm{ml} \mathrm{X-Gal)} 40 \mathrm{mM}$ citrate/phosphate buffer ( $\mathrm{pH} 6.0$ ), $5 \mathrm{mM}$ potassium ferrocyanide, $5 \mathrm{mM}$ potassium ferricyanide, $150 \mathrm{mM} \mathrm{NaCl}$, and $2 \mathrm{mM}$
$\mathrm{MgCl}_{2}$ ) in a chamber maintained at $37^{\circ} \mathrm{C}$ for $12 \mathrm{~h}$. The degree of senescence-associated cells was calculated as a percentage of the total number of cells.

RNA isolation and RT-PCR. Chondrocytes $\left(1 \times 10^{6}\right.$ cells $\left./ \mathrm{cm}^{2}\right)$ were grown in $60-\mathrm{mm}$ culture dishes, and incubated for $24 \mathrm{~h}$ in fresh medium with or without cilostazol. Next, total RNA was isolated using TRIzol reagent and reverse transcription was performed using superscript reverse transcriptase (Invitrogen, Carlsbad, CA, USA) according to the manufacturer's instructions. Total RNA ( $2 \mu \mathrm{g})$ was used to prepare cDNA. The following primers were used in our study: type I collagen, forward, 5'-GACCCAAAGGTTCTCGTGGT-3' and reverse, 5'-CTTTCTCCTCTCTGACCGGG-3'; type II collagen, forward 5'-GGTAAGTGGGGCAAGACCAT-3' and reverse 5'-TTTTGCAGTCTGCCCAGTTC-3'; glyceraldehyde 3-phosphate dehydrogenase (GAPDH), forward, 5'-TGAAGGTCGGAGTCAACGGATTTGGT-3' and reverse, 5'-CATGTGGGCCATGAGGTCCACCAC-3'. PCR reactions in $25 \mu 1$ reaction volumes were performed as follows: $95^{\circ} \mathrm{C}$ for $5 \mathrm{~min}, 30 \mathrm{cycles}$ of $94^{\circ} \mathrm{C}$ for $30 \mathrm{sec}$, either $52^{\circ} \mathrm{C}$ (type I collagen) or $56^{\circ} \mathrm{C}$ (type II collagen) for $30 \mathrm{sec}, 72^{\circ} \mathrm{C}$ for $1 \mathrm{~min}$, and $72^{\circ} \mathrm{C}$ for $5 \mathrm{~min}$.

Western blot analysis. Equivalent amounts $(20 \mu \mathrm{g})$ of total proteins were loaded onto $12 \%$ sodium dodecyl sulphate (SDS)-polyacrylamide gels for electrophoresis. The proteins were then transferred onto a nitrocellulose membrane by using an electroblotting apparatus (Bio-Rad, Richmond, $\mathrm{CA}$ ), and the membranes were incubated with each primary antibody. The blots were washed with TBS-T and incubated with an HRP-conjugated secondary anti-rabbit antibody. The membranes were developed using the ECL reaction system and visualized using the LAS-3000 Luminescent Image Analyzer (FujiFilm, Japan). Image Gauge Ver. 3.0 software was used to calculate the changes in protein expression, and $\beta$-actin was used as an internal control to ensure equal protein sample loading.

Immunofluorescence staining. Chondrocytes were cultured on collagen-coated 4-well glass chamber slides for $48 \mathrm{~h}$ in DMEM containing 10\% FBS. Cells were fixed in 4\% paraformaldehyde/ PBS, followed by permeabilization in $1 \%$ Triton X-100/PBS. Appropriate primary antibodies were added to the cells for $30 \mathrm{~min}$ at $37^{\circ} \mathrm{C}$. For secondary labeling, cells were incubated with FITC-conjugated secondary antibody (Invitrogen) for $30 \mathrm{~min}$ at $37^{\circ} \mathrm{C}$. Nuclei were counterstained using propidium iodide (PI). Fluorescent images were observed and analyzed using a laser-scanning confocal microscope.

Statistics or reproducibility. Each experiment was repeated at least 3 times. Data were expressed as the means \pm SE from each independent experiment. The data for the experimental and control groups were tested for statistical significance by one-tailed Student's $t$ test, with $\mathrm{P}<0.05$ accepted as the level of significance.

\section{Results}

Cilostazol induces dedifferentiation in primary rat articular chondrocytes. To determine the role of cilostazol in the 
A
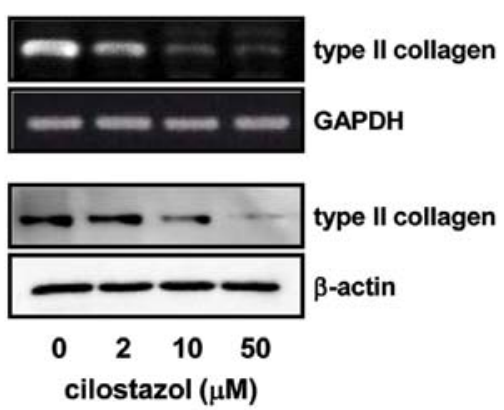

C

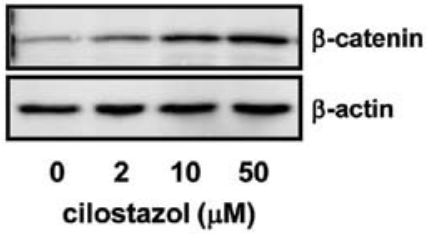

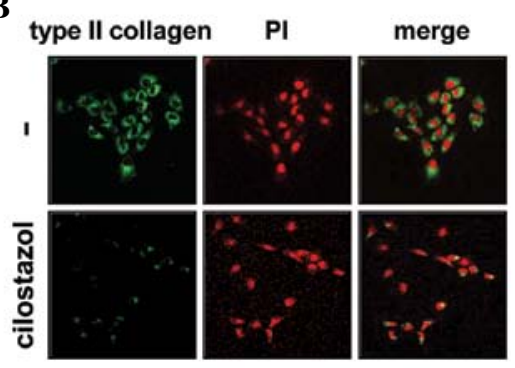

D

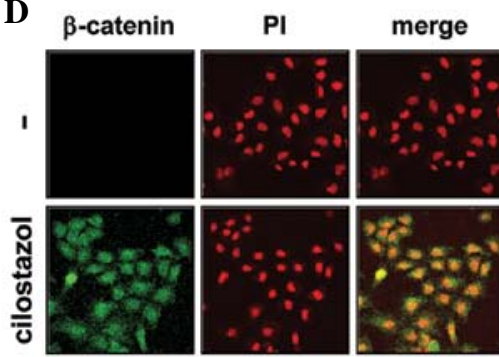

Figure 1. Effect of cilostazol on the expression of phenotypic markers in chondrocytes. Rat articular chondrocytes were treated with different concentrations of cilostazol for $24 \mathrm{~h}$. (A) Levels of type I and II collagen were analyzed by RT-PCR and western blot analysis. GAPDH was used as an internal control. $\beta$-actin was used as an internal control to ensure equal protein loading. (B) The cells were fixed and reacted with type II collagen antibody for immunofluorescence staining by confocal microscopy. The right panels show superimposed $\beta$-catenin antibody and propidium iodide (PI) immunoreactivity (orange). (C) Western blot analysis with $\beta$-catenin antibody. (D) Immunofluorescence staining with $\beta$-catenin antibody.

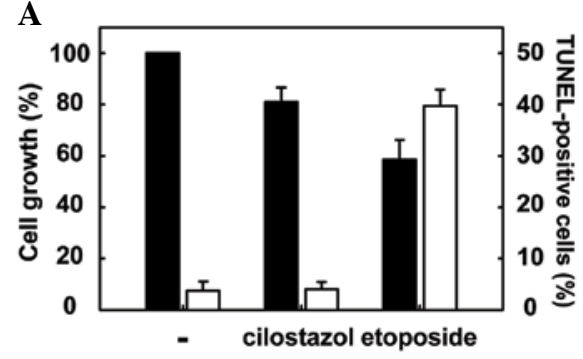

B
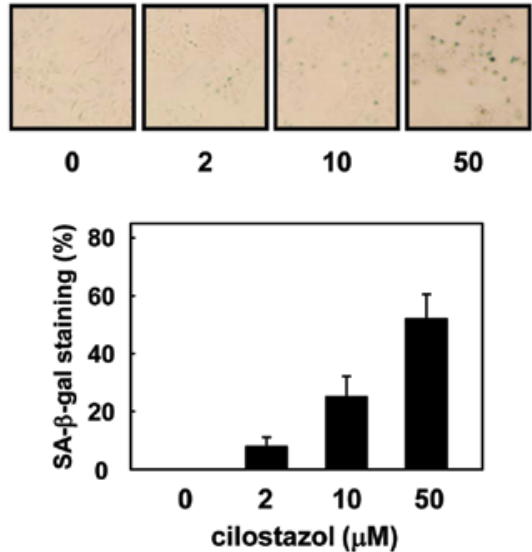

Figure 2. Effect of cilostazol on cellular senescence in articular chondrocytes. (A) Rat articular chondrocytes were treated with $50 \mu \mathrm{M}$ cilostazol or $200 \mu \mathrm{g} / \mathrm{ml}$ etoposide for $24 \mathrm{~h}(\boldsymbol{\bullet}$, total cells; $\square$, apoptotic cells). The cells were assessed for apoptosis by TUNEL assay. TUNEL-positive cells were counted under a confocal microscope; 250-300 cells were counted for each condition. Three independent experiments were performed, and values are represented as means \pm SD from the 3 experiments. (B) Rat chondrocytes were treated with different concentrations of cilostazol for $48 \mathrm{~h}$ and stained for SA- $\beta$-gal activity; representative images were obtained at x 20 magnification. Percentages of SA- $\beta$-gal-positive cells were determined from the numbers of blue cells per 200 cells in a randomly selected area.

maintenance of chondrocyte phenotypes, we first investigated the effect of cilostazol on the expression of type II collagen in chondrocytes. Primary rat articular chondrocytes were isolated, maintained, and treated with various concentrations $(2,10$, and $50 \mu \mathrm{M}$ ) of cilostazol for $24 \mathrm{~h}$. As shown in Fig. 1A and B, cilostazol significantly reduced the expression of type II collagen, which plays a crucial role in regulating chondrocyte functions by facilitating cell-matrix interactions. In addition, cilostazol stimulated the accumulation of $\beta$-catenin, a phenotypic marker for the differentiation and dedifferentiation of chondrocytes
(Fig. 1C and D). These results suggested that cilostazol causes cellular dedifferentiation, i.e., loss of differentiated chondrocyte phenotypes, in primary articular chondrocytes.

Cilostazol-induced dedifferentiation enhances cellular senescence in chondrocytes. Next, we assessed whether the cilostazol-induced dedifferentiation is associated with cellular senescence in primary chondrocytes. Cilostazol decreased cell proliferation without affecting cell viability (Fig. 2A). P0 cells were treated with various concentrations $(2,10$, and $50 \mu \mathrm{M})$ of 
A
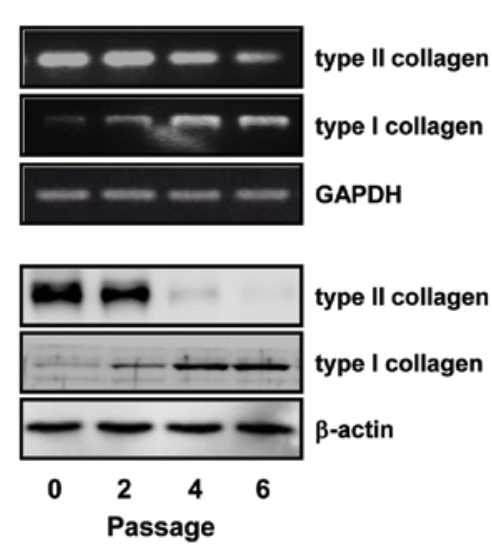

B

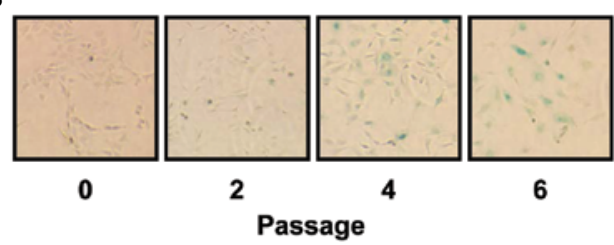

C

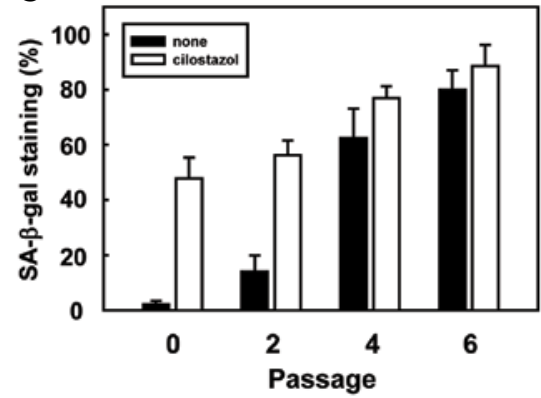

Figure 3. Effect of cilostazol on subculture-induced dedifferentiation of chondrocytes. Primary articular chondrocytes were serially subcultured up to 6 passages. (A) Whole cell extracts were analyzed by RT-PCR, and western blot analysis was performed using appropriate primers and antibodies, as indicated. GAPDH and $\beta$-actin were used as internal controls for the RT-PCR and western blot analysis, respectively. (B) Chondrocyte senescence was confirmed using a SA- $\beta$-gal activity assay. (C) P0, P2, P4, and P6 cells were treated with $50 \mu \mathrm{M}$ cilostazol for $48 \mathrm{~h}$, and cellular senescence was assessed by SA- $\beta$-gal staining. SA- $\beta$-gal-positive cells were counted under a microscope; 200 cells were counted in a randomly selected area.

A TUNEL PI merge

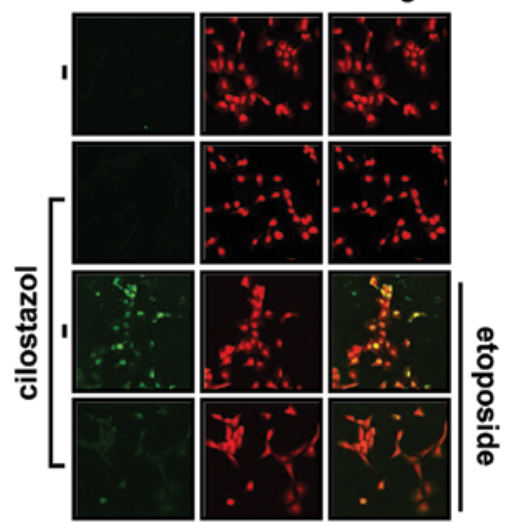

B

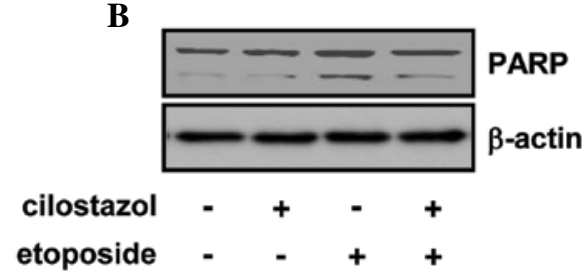

C TUNEL PI merge

PO
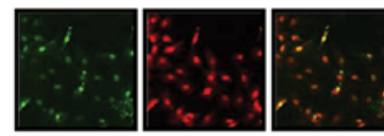

P6
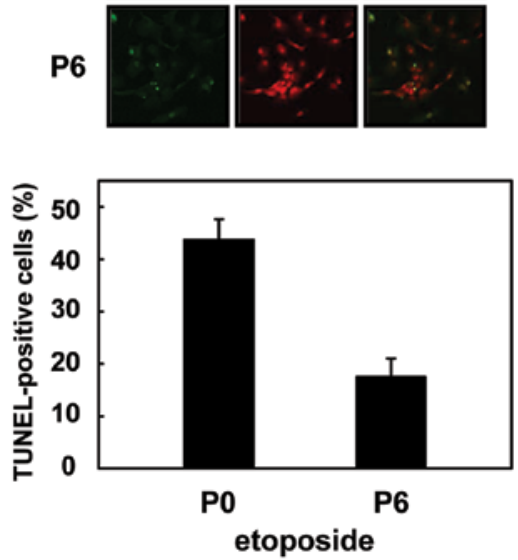

Figure 4. Effect of cilostazol on the etoposide-induced apoptosis in chondrocytes. Rat chondrocytes were pretreated with $50 \mu \mathrm{M}$ cilostazol for $48 \mathrm{~h}$, and then incubated with $200 \mu \mathrm{g} / \mathrm{ml}$ etoposide for another $24 \mathrm{~h}$. (A) The cells were assessed for apoptosis by TUNEL assay. The merged images, resulting from the overlap of TUNEL-positive (green) and PI labeled (red) areas, were observed in the cells. (B) Cell lysates were analyzed by western blot analysis to detect the level of PARP cleavage (C) P0 and P6 cells were treated with $200 \mu \mathrm{g} / \mathrm{ml}$ etoposide for $24 \mathrm{~h}$, and apoptosis was assessed by TUNEL assay. TUNEL-positive cells were counted under a confocal microscope; 250-300 cells were counted for each condition.

cilostazol for $48 \mathrm{~h}$. Then, the induction of cellular senescence was confirmed by an increase in SA- $\beta$-gal-positive cells in cilostazol-treated chondrocytes compared to those in the control. Interestingly, cilostazol-treated chondrocytes showed significant increase in specific SA- $\beta$-gal staining (Fig. 2B).
Dedifferentiated chondrocytes are sensitive to cellular senescence by cilostazol. To investigate whether cellular senescence is associated with the differentiation or dedifferentiation status of chondrocytes, we determined the effect of cilostazol on cellular senescence during subculture-induced 
dedifferentiation of chondrocytes. Primary chondrocytes were serially subcultured to induce cellular dedifferentiation. As shown in Fig. 3A, the expression of type I and type II collagens was significantly increased and decreased in P4 and P6 cells, respectively. The expression of $\beta$-catenin showed a similar pattern as type II collagen (data not shown). Importantly, serial subculture of chondrocytes also induced changes in senescent phenotypes, such as changes in cell morphology, decreases in cell proliferation, and increases in specific SA- $\beta$-gal staining (Fig. 3B). This suggests that chondrocyte senescence also increased during subculture-induced dedifferentiation.

Next, we observed cellular senescence in chondrocytes at different passages ( $\mathrm{P} 0, \mathrm{P} 2, \mathrm{P} 4$, and P6) by subculture. Dedifferentiated chondrocytes showed significant cellular senescence that increased with passage number. This suggested that dedifferentiated chondrocytes slowly and spontaneously progressed to cellular senescence. Cilostazol significantly increased the SA- $\beta$-gal staining of $\mathrm{P} 2$ cells, and slightly increased the SA- $\beta$-gal staining of P4 and P6 cells (Fig. 3C). Cilostazol had a weak effect on senescence in dedifferentiated chondrocytes at high passage number. This is because with increasing passage number increases, senescence in cells increases. Taken together, these results suggest that senescence in chondrocytes is associated with the dedifferentiation status, which indicates the loss of differentiated chondrocyte phenotypes.

Cilostazol renders chondrocytes resistant to etoposide-induced apoptosis via cellular senescence. TUNEL assay was used to determine whether cilostazol-induced dedifferentiation and senescence changed the susceptibility of articular chondrocytes against apoptosis mediated by an apoptosis-inducing drug. Cells were pretreated with cilostazol for $48 \mathrm{~h}$, and then stimulated with $200 \mu \mathrm{g} / \mathrm{ml}$ etoposide for an additional $24 \mathrm{~h}$. The percentage of TUNEL-positive cells was markedly reduced when chondrocytes were treated with cilostazol and etoposide compared to when they were treated with etoposide alone (Fig. 4A). Etoposide is known to induce poly(ADP-ribose) polymerase (PARP) cleavage, and etoposide-induced PARP cleavage was reduced in cilostazol-treated cells (Fig. 4B). To show that senescent cells are more resistant than young cells, we used P0 and senescent P6 cells. As shown in Fig. 4C, when cells were stimulated with etoposide for $24 \mathrm{~h}, \sim 45$ and $19 \%$ of P0 and P6 cells were TUNEL-positive cells, respectively. These results suggest that cilostazol renders chondrocytes resistant to the apoptosis inducer by inducing cellular senescence.

\section{Discussion}

Chondrocyte apoptosis plays an important role in the degeneration and degradation of articular cartilage in the cases of osteoarthritis (OA) $(22,23)$ and rheumatoid arthritis $(7,24)$. We previously showed that cilostazol protects rat chondrocytes against $\mathrm{NO}$-induced apoptosis in vitro and prevents cartilage destruction in mono-iodoacetate (MIA)-induced OA in a rat model expressing inducible NO synthase (iNOS) (19). Recent evidence suggests that cilostazol inhibits apoptosis under various conditions (25-27). This raises a possibility that cilostazol can be used for treating diseases associated with apoptotic cell death. Therefore, the precise mechanism underlying the cilostazol mediated maintenance and induction of cell death in chondrocytes of articular cartilage needs to be elucidated.

In the present study, we showed that cilostazol accelerates cellular dedifferentiation as well as cellular senescence in primary rat articular chondrocytes. This finding is supported by the following observations. As shown in Fig. 1, cilostazol significantly reduced the expression of type II collagen and stimulated the accumulation of $\beta$-catenin, which are typical phenotypic markers of chondrocyte differentiation and dedifferentiation $(5,28-30)$. This suggests that cilostazol induces cellular dedifferentiation in primary articular chondrocytes. To confirm this finding, we also analyzed the changes in the levels of phenotypic markers during subculture-induced dedifferentiation of chondrocytes. The expression of type II collagen was completely abolished and that of type I collagen was significantly increased in P4 and P6 cells. There was no increase in the level of type I collagen in cilostazol-induced dedifferentiated chondrocytes. However, serial subculture of primary chondrocytes resulted in a decrease of cell proliferation causing changes in cell morphology in a passage-dependent manner. Therefore, we thought that the dedifferentiated state of chondrocytes could be related to cellular senescence in cilostazol-treated or subculture-induced chondrocytes.

Cellular senescence refers to a state when somatic cells enter a state of permanent growth arrest, resulting in progressive functional decline and eventual death. Senescent cells are characterized by an enlarged, flattened morphology and SA- $\beta$-gal expression $(21,31)$. Senescent cells remain metabolically active and are resistant to apoptosis induced by exposure to genotoxic stress for a long period (32). Chondrocyte senescence causes a decline in chondrocyte numbers due to apoptotic cell death and is important in the development and progression of OA $(33,34)$. In fact, senescent chondrocytes accumulate with age or in the cases of OA in the articular cartilage $(9,13,14)$. Therefore, we investigated cellular senescence by conducting SA- $\beta$-gal staining assay in cilostazol-treated chondrocytes. As shown in Fig. 2, there was a significant increase in SA- $\beta$-gal staining in chondrocytes treated with cilostazol. In addition, etoposideinduced apoptosis was also reduced in cilostazol-treated or subculture-induced chondrocytes.

In conclusion, our results suggest that cilostazol induces cellular dedifferentiation and senescence in rat articular chondrocytes and render them resistant to apoptosis induced by genotoxic stress. Further studies are needed to clarify the in vivo effects of cilostazol on the dedifferentiation and senescence of chondrocytes in the articular cartilage of cilostazol-treated rats.

\section{Acknowledgements}

This work was supported by the Korea Science and Engineering Foundation (grant no. R01-2007-000-20100-0).

\section{References}

1. Sandell LJ and Adler P: Developmental patterens of cartilage. Front Biosci 4: D731-D742, 1999.

2. DeLise AM, Fischer L and Tuan RS: Cellular interactions and signaling in cartilage development. Osteoarthritis Cartilage 8: 309-334, 2000 .

3. Goldring MB: The role of the chondrocyte in osteoarthritis. Arthritis Rheum 43: 1916-1926, 2000. 
4. Roughley PJ: Articular cartilage and changes in arthritis: noncollagenous proteins and proteoglycans in the extracellular matrix of cartilage. Arthritis Res 3: 342-347, 2001.

5. Eyre D: Collagen of articular cartilage. Arthritis Res 4: 30-35, 2002.

6. Cawston T, Billington C, Cleaver C, Elliott S, Hui W, Koshy P, Shingleton B and Rowan A: The regulation of MMPs and TIMPs in cartilage turnover. Ann NY Acad Sci 878: 120-129, 1999.

7. Kim HA and Song YW: Apoptotic chondrocyte death in rheumatoid arthritis. Arthritis Rheum 42: 1528-1537, 1999.

8. Aigner T and Kim HA: Apoptosis and cellular vitality: issues in osteoarthritic cartilage degeneration. Arthritis Rheum 46: 1986-1996, 2002.

9. Martin JA and Buckwalter JA: Telomere erosion and senescence in human articular cartilage chondrocytes. J Gerontol A Biol Sci Med Sci 56: B172-B179, 2001.

10. Asada S, Fukuda K, Nishisaka F, Matsukawa M and Hamanisi C: Hydrogen peroxide induces apoptosis of chondrocytes; involvement of calcium ion and extracellular signal-regulated protein kinase. Inflamm Res 50: 19-23, 2001.

11. Itahana K, Campisi J and Dimri GP: Mechanisms of cellular senescence in human and mouse cells. Biogerontology 5: 1-10, 2004.

12. Wlaschek M, Ma W, Jansen-Dürr P and Scharffetter-Kochanek K Photoaging as a consequence of natural and therapeutic ultraviolet irradiation: studies on PUVA-induced senescence-like growth arrest of human dermal fibroblasts. Exp Gerontol 38: 1265-1270, 2003.

13. Loeser RF: Aging and osteoarthritis: the role of chondrocyte senescence and aging changes in the cartilage matrix. Osteoarthritis Cartilage 17: 971-979, 2009.

14. Martin JA and Buckwalter JA: Aging, articular cartilage chondrocyte senescence and osteoarthritis. Biogerontology 3: 257-264, 2002.

15. Kimura Y, Tani T, Kanbe $\mathrm{T}$ and Watanabe K: Effect of cilostazol on platelet aggregation and experimental thrombosis. Arzneimittelforschung 35: 1144-1149, 1985.

16. Tanaka K, Gotoh F, Fukuuchi Y, Amano T, Uematsu D, Kawamura J, Yamawaki T, Itoh N, Obara K and Muramatsu K: Effects of a selective inhibitor of cyclic AMP phosphodiesterase on the pial microcirculation in feline cerebral ischemia. Stroke 20: 668-673, 1989.

17. Jaff KR: Pharmacotherapy for peripheral arterial disease: emerging therapeutic options. Angiology 53: 627-633, 2002.

18. Dawson DL and Cutler BS: Meissner MH and Strandness DE Jr: Cilostazol has beneficial effects in treatment of intermittent claudication: results from a multicenter, randomized, prospective double-blind trial. Circulation 98: 678-686, 1998.

19. Lee SW, Song YS, Shin SH, Kim KT, Park YC, Park BS, Yun I, Kim K, Lee SY, Chung WT, et al: Cilostazol protects rat chondrocytes against nitric oxide-induced apoptosis in vitro and prevents cartilage destruction in a rat model of osteoarthritis. Arthritis Rheum 58: 790-800, 2008.

20. Park SY, Lee SW, Shin HK, Chung WT, Lee WS, Rhim BY, Hong KW and Kim CD: Cilostazol enhances apoptosis of synovial cells from rheumatoid arthritis patients with inhibition of cytokine formation via Nrf2-linked heme oxygenase 1 induction. Arthritis Rheum 62: 732-741, 2010.
21. Dimri GP, Lee X, Basile G, Acosta M, Scott G, Roskelley C, Medrano EE, Linskens M, Rubelj I and Pereira-Smith O: A biomarker that identifies senescent human cells in culture and in aging skin in vivo. Proc Natl Acad Sci USA 92: 9363-9367, 1995 .

22. Hashimoto S, Ochs RL, Komiya S and Lotz M: Linkage of chondrocyte apoptosis and cartilage degradation in human osteoarthritis. Arthritis Rheum 41: 1632-1638, 1998.

23. Goggs R, Carter SD, Schulze-Tanzil G, Shakibaei M and Mobasheri A: Apoptosis and the loss of chondrocyte survival signals contribute to articular cartilage degradation in osteoarthritis. Vet J 166: 140-158, 2003.

24. Yatsugi N, Tsukazaki T, Osaki M, Koji T, Yamashita S and Shindo H: Apoptosis of articular chondrocytes in rheumatoid arthritis and osteoarthritis: correlation of apoptosis with degree of cartilage destruction and expression of apoptosis-related proteins of p53 and c-myc. J Orthop Sci 5: 150-156, 2000.

25. Lee SW, Lee HJ, Chung WT, Choi SM, Rhyu SH, Kim DK, Kim KT, Kim JY, Kim JM and Yoo YH: TRAIL induces apoptosis of chondrocytes and influences the pathogenesis of experimentally induced rat osteoarthritis. Arthritis Rheum 50: 534-542, 2004.

26. Kim KY, Shin HK, Choi JM and Hong KW: Inhibition of lipopolysaccharide-induced apoptosis by cilostazol in human umbilical vein endothelial cells. J Pharmacol Exp Ther 300: 709-715, 2002

27. Choi JM, Shin HK, Kim KY, Lee JH and Hong KW: Neuroprotective effect of cilostazol against focal cerebral ischemia via antiapoptotic action in rats. J Pharmacol Exp Ther 300: 787-793, 2000.

28. Aigner T, Zhu Y, Chansky HH, Matsen FA III, Maloney WJ and Sandell LJ: Reexpression of type IIA procollagen by adult articular chondrocytes in osteoarthritic cartilage. Arthritis Rheum 42: 1443-1450, 1999.

29. Henrotin Y, Addison S, Kraus V and Deberg M: Type II collagen markers in osteoarthritis: what do they indicate? Curr Opin Rheumatol 19: 444-450, 2007.

30. Yoon YM, Kim SJ, Oh CD, Ju JW, Song WK, Yoo YJ, Huh TL and Chun JS: Maintenance of differentiated phenotype of articular chondrocytes by protein kinase $\mathrm{C}$ and extracellular signal-regulated protein kinase. J Biol Chem 277: 8412-8420, 2002.

31. Brandl A, Hartmann A, Bechmann V, Graf B, Nerlich M and Angele P: Oxidative stress induces senescence in chondrocytes. J Orthop Res 29: 1114-1120, 2011.

32. Salminen A, Ojala J and Kaarniranta K: Apoptosis and aging: increased resistance to apoptosis enhances the aging process. Cell Mol Life Sci 68: 1021-1031, 2011.

33. Horton WE Jr, Feng L and Adams C: Chondrocyte apoptosis in development, aging and disease. Matrix Biol 17: 107-115, 1998.

34. Kuhn K, D'Lima DD, Hashimoto S and Lotz M: Cell death in cartilage. Osteoarthritis Cartilage 12: 1-16, 2004. 